\title{
Double chirp-taper x-ray free-electron laser for attosecond pump-probe experiments
}

\author{
Zhen Zhang, Joseph Duris, James P. MacArthur, Zhirong Huang, and Agostino Marinelli* \\ SLAC National Accelerator Laboratory, Menlo Park, California 94025, USA
}

(Received 6 February 2019; published 2 May 2019)

\begin{abstract}
In this paper we propose a double chirp-taper method to produce two subfemtosecond x-ray freeelectron lasers (XFELs) using electron beams with sinusoidal energy modulation. The taper of the undulator is optimized to control the power profile of each XFEL pulse to obtain a single-spike power shape. We present start-to-end numerical simulations of this method to demonstrate the generation of two XFEL pulses with $\sim 0.4 \mathrm{fs}$ full width at half maximum, with a time separation tunable from 0 to tens of fs and energy separation limited by the available undulator tuning range. The output of this method provides a powerful tool for XFEL pump-probe experiments at the attosecond timescale.
\end{abstract}

DOI: 10.1103/PhysRevAccelBeams.22.050701

\section{INTRODUCTION}

X-ray free-electron lasers (XFELs) are routinely used for resolving dynamical processes in molecules and materials at the femtosecond timescale [1,2]. While much progress has been made with femtosecond XFELs, the ability to access subfemtosecond (sub-fs) timescales would enable an entirely new class of experiments (see, e.g., [3]). In fact, the typical timescale for valence electronic motion is hundreds of attoseconds [4], which means that time-resolved experiments at the femtosecond timescale can resolve atomic motion but they are blind to the motion of electrons. The ability to understand electronic motion at the relevant timescale is critical to our understanding of atomic and molecular physics [5] and, ultimately, chemistry [6].

The path to sub-fs time-resolution requires not only the generation of sub-fs pulses, but also the ability to synchronize two or more pulses of different energies with attosecond precision. Two-color FEL schemes have been demonstrated and successfully applied to a wide range of experiments in the femtosecond regime. Two-color FELs typically have better arrival time stability compared to $\mathrm{x}$-ray pump/probe schemes that rely on external lasers. This is because both colors are either emitted by the same electron bunch [7-10] or by two electron bunches generated within the same rf bucket [11]. This, in turn, effectively decouples the relative timing jitter of the two pulses from the absolute arrival time jitter of the electron beam. Timing stability of a

\footnotetext{
*marinelli@slac.stanford.edu
}

Published by the American Physical Society under the terms of the Creative Commons Attribution 4.0 International license. Further distribution of this work must maintain attribution to the author(s) and the published article's title, journal citation, and DOI. few fs or better is routinely achieved in two-color experiments (see, e.g., [12]).

In this paper we propose a method to generate pairs of sub-fs $x$-ray pulses with attosecond timing stability and controllable separation based on a double chirp/taper scheme driven by self-modulation in a magnetic wiggler technique $[13,14]$. Since this method relies on variable-gap undulators to generate two independent FEL pulses, the energy of the two pulses can be varied arbitrarily, allowing for pump/probe experiments at different atomic sites and enabling the study of charge and energy transfer in molecules at the attosecond timescale.

Our scheme is an extension of previous work on chirptapered FELs [15-20]. An electron beam with a large energy modulation travels through a tapered undulator. If the energy modulation is larger than the FEL energy bandwidth and the chirp-taper matching condition is satisfied, lasing is only supported on a short fraction of the electron bunch (namely the fraction where the local energy chirp is matched to the undulator taper). By dividing the undulator in two parts and using two different tapers, one can lase independently on two different slices of the electron bunch, generating two independent pulses. A magnetic chicane placed between the two undulators can be used to delay the second pulse with respect to the first with sub-fs precision, allowing for continuous control of the delay between the two pulses.

While this scheme can be realized with any beam modulation technique, in this paper we analyze in detail the case of a two-stage self-modulation scheme. This method was recently developed at LCLS and it uses coherent undulator radiation emitted by the tail of the bunch as an infrared seed to generate a quasisingle cycle modulation with carrier-envelope phase stability $[13,14]$.

This method allows for sub-fs timing stability while, at the same time, allowing full saturation and full time delay 
tunability through zero delay. Compared to previous methods our proposal offers a way to generate sub-fs pulses and combines the excellent timing stability of the split undulator $[7,9]$ with the peak power and timing tunability of the freshslice method [10]. Furthermore, the use of a passive modulator makes this method scalable to high-repetition rate machines, enabling attosecond pump/probe at the next generation of superconducting XFELs.

The remainder of the paper is organized as follows. In Sec. II, simulations with an ideal electron beam that has a sinusoidal energy modulation are performed to demonstrate the proposed method. And then a start-to-end simulation with the LCLS-II copper linac beamline parameters, including the self-modulation in the wiggler, is presented in the following section. Some discussions about the proposed method are presented in Sec. IV and a short summary is given in Sec. V.

\section{SIMULATIONS WITH IDEAL BEAM}

In this section, we illustrate the double chirp-taper method described above with numerical simulations assuming an idealized beam. Specifically, we use a beam with no variations in current, energy spread, and emittance along the longitudinal direction. The main parameters are shown in Table I and the longitudinal phase space of the electron beam is presented in Fig. 1. A sinusoidal energy modulation with $\lambda_{m}=2 \mu \mathrm{m}$ period and $\Delta \gamma= \pm 50$ amplitude is added onto the electron beam. For the beam with sinusoidal energy modulation, the maximum slope of the energy chirp can be estimated by the modulation period and amplitude

$$
\left|\frac{d \gamma}{d s}\right|_{\max }=\frac{2 \pi}{\lambda_{m}} \Delta \gamma
$$

The chirp-taper matching condition [17,21-23] can be written as

$$
\frac{\lambda_{u} K(0)}{1+K(0)^{2} / 2} \frac{d K}{d z}=\frac{2 \lambda_{r}}{c \gamma_{r}} \frac{d \gamma}{d t}
$$

where $K(0)$ is the undulator strength at the beginning of each section, $\gamma_{r}$ the resonance beam energy and $c$ the speed

TABLE I. Parameters of the electron beam and undulators.

\begin{tabular}{lcc}
\hline \hline Parameter & Value & Unit \\
\hline Beam energy $\gamma$ & 9500 & \\
Slice energy spread $\sigma_{\gamma}$ & 8 & \\
Normalized emittance $\varepsilon_{N}$ & 0.5 & $\mu \mathrm{m}$ \\
Beam current $I$ & 4.5 & $\mathrm{kA}$ \\
Modulation period $\lambda_{m}$ & 2 & $\mu \mathrm{m}$ \\
Modulation amplitude $\Delta \gamma$ & 50 & \\
Photon energy $h \nu$ & $\sim 900 / 1100$ & $\mathrm{eV}$ \\
Undulator period $\lambda_{u}$ & 3 & $\mathrm{~cm}$ \\
Undulator strength $K^{\mathrm{a}}$ & $3.82 / 3.41$ & \\
\hline \hline
\end{tabular}

${ }^{a}$ Values at the beginning of taper.

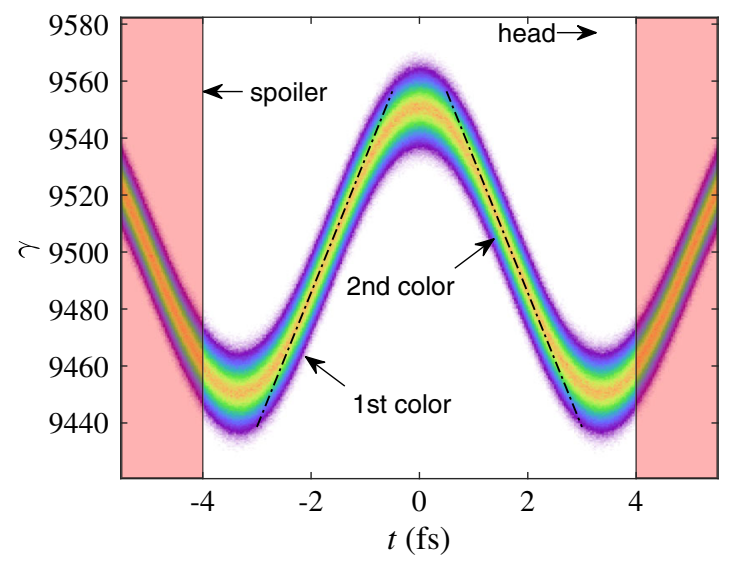

FIG. 1. Longitudinal phase space of the ideal electron beam. The shaded area is spoiled to suppress lasing in the undulator. The dotted lines denote the peak slopes of the energy chirp. The left slope is used to generate the first color and the right one is for the second color. The beam's head is to the right.

of light. If the correlated energy spread in a cooperation length (i.e., the slippage length accrued in a gain-length), is larger than the FEL bandwidth then lasing can only happen in the regions of the bunch where Eq. (2) is verified. By splitting the undulator in two parts with different taper amplitudes, we can enable lasing on two independent slices of the electron bunch (namely the ones with the maximum positive and negative chirps), therefore generating two independent $\mathrm{x}$-ray pulses.

The layout of the double-taper scheme is shown in Fig. 2, with the undulator divided in two parts separated by a magnetic chicane. The time delay between the two pulses is given roughly by:

$$
\Delta t= \pm \frac{\lambda_{\bmod }}{2 c}+\delta t_{s}+\delta t_{C}
$$

where $\lambda_{\text {mod }}$ is the modulation wavelength, $\delta t_{s}$ is the slippage length in the second undulator, and $\delta t_{C}$ is the time-delay introduced by the magnetic chicane. The \pm sign ambiguity is due to the possibility of choosing either the positively or negatively chirped parts of the bunch to emit the first color. To enable scanning through zero delay it is convenient to generate the first pulse with the tail of the electron bunch and the second pulse with the head. In this way the initial delay (i.e., the delay when the chicane is off) is negative and the total delay can be increased to positive values by increasing $\delta t_{C}$.

To accomplish this the left energy chirp with positive slope (with reference to Fig. 1) is used to generate the first color in an undulator section with gradually increasing strength while the right one with negative slope will lase in the second undulator section with gradually decreasing strength. We note that for the delay to be repeatable the phase of the modulation needs to be stable, which means 


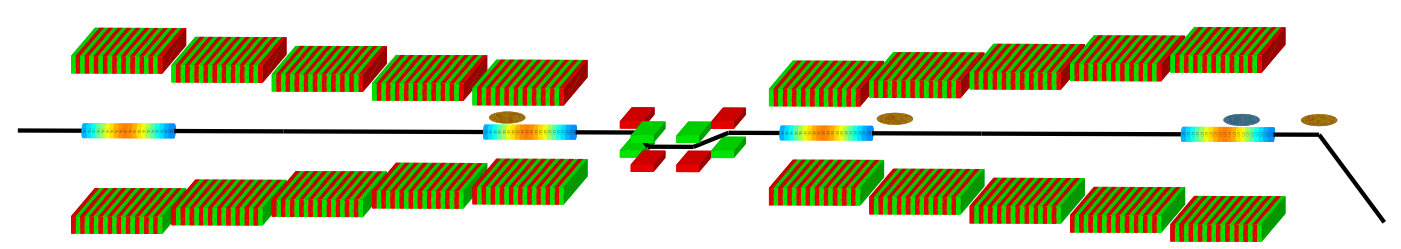

FIG. 2. The layout of the double-taper scheme to generate sub-fs two-color FEL pulses, including two undulator sections with opposite taper directions and a magnetic chicane between them. The colors in the beam represent the beam energy distribution.

that a carrier-envelope phase stable modulator is needed for this scheme to work.

The energy separation of the two colors is limited by the tunable range of the undulator strength, which allows $100 \%$ energy separation of the colors. In this paper the photon energies of the two colors are $\sim 900 \mathrm{eV}$ and $\sim 1100 \mathrm{eV}$.

Based on Eq. (2) we can estimate and optimize the taper with the aim of maximizing peak power and achieving single-spike lasing. All simulations in this paper are conducted by the three-dimensional, time-dependent code GENESIS [24]. As the energy separation of the two colors $(200 \mathrm{eV})$ is much larger than the FEL bandwidth, we have to simulate FEL lasing process separately in the two sections. The input electron beam of the second section is the output beam after lasing in the first section. In the simulations as shown in Fig. 1, we spoil the beam quality of the neighboring modulation periods to suppress the lasing from those parts which also have matched energy chirp with the taper. Experimentally this can be achieved either with an emittance-spoiling foil in the magnetic chicane $[25,26]$ or by shaping the temporal shape of the laser heater [27]. The latter option is the preferred method for highrepetition rate FELs.

Using the electron beam in Fig. 1, the optimized undulator taper for two-color generation is presented in Fig. 3. The undulator strength $K$ at the beginning of the taper is 3.82 for the $\sim 900 \mathrm{eV}$ FEL pulse and 3.41 for the $\sim 1100 \mathrm{eV}$ pulse. The optimized slopes of the taper $d K /(K d z)$ are $2.4 \times 10^{-3}$ for the first section and $-1.8 \times 10^{-3}$ for the
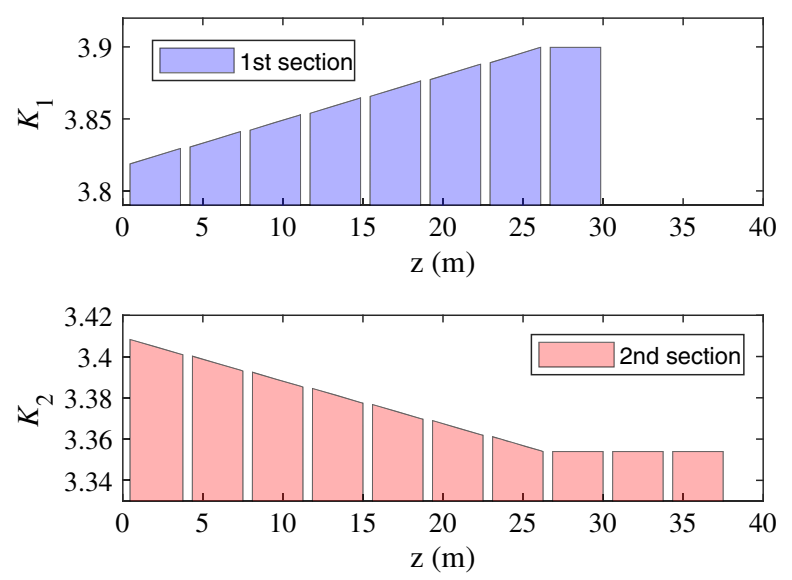

FIG. 3. The optimized undulator strength taper of the two sections for the generation of two-color FELs. second section, which are very close to the corresponding peak taper coefficients estimated by Eqs. (1) and (2), $2.5 \times 10^{-3}$ for $900 \mathrm{eV}$ and $-1.9 \times 10^{-3}$ for $1100 \mathrm{eV}$. The undulator settings of the two sections are shown in Fig. 3. For each section, the end of the undulator has a uniform strength. This allows further amplification of the FEL power after saturation, as the FEL pulse moves toward the neighboring slices of the beam which have zero chirp [28]. Figure 4 shows the power profiles of a typical FEL pulse from the fifth to eighth undulator in the first section. Since the undulator taper matches the peak slope of the energy chirp, the FEL power will first accumulate around the matched part of the electron beam. As the pulse is amplified it also slips forward with respect to the beam. As the pulse approaches saturation the pulse duration is also shortened thanks to the well-known superradiant behavior of short-pulse FELs close to saturation [29-31].

One of the most important considerations in the taper optimization is to achieve one isolated pulse for each color. The strategy of matching the taper to the maximum energy chirp guarantees that most shots produce single-spike FEL pulses. Figure 5 gives examples of the power profile with single, double, or multiple spikes and their corresponding proportion for a series of simulations. Note that the two pulses overlap together if the delay of the chicane between the undulators is zero so as to illustrate the two pulses clearly, an additional 5 fs delay from the chicane is added to the $1100 \mathrm{eV}$ pulse. The statistical result is obtained over

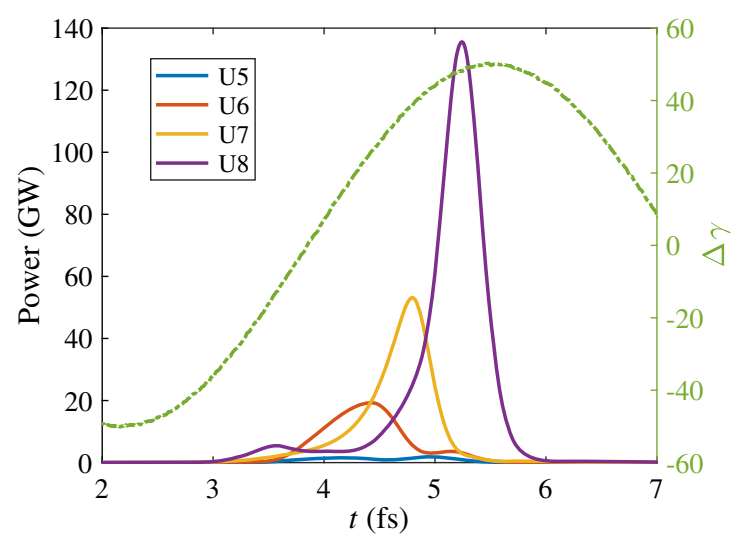

FIG. 4. Power profiles of one typical FEL pulse along the first undulator section. The dotted line shows the energy deviation along the longitudinal coordinate of the electron beam. 

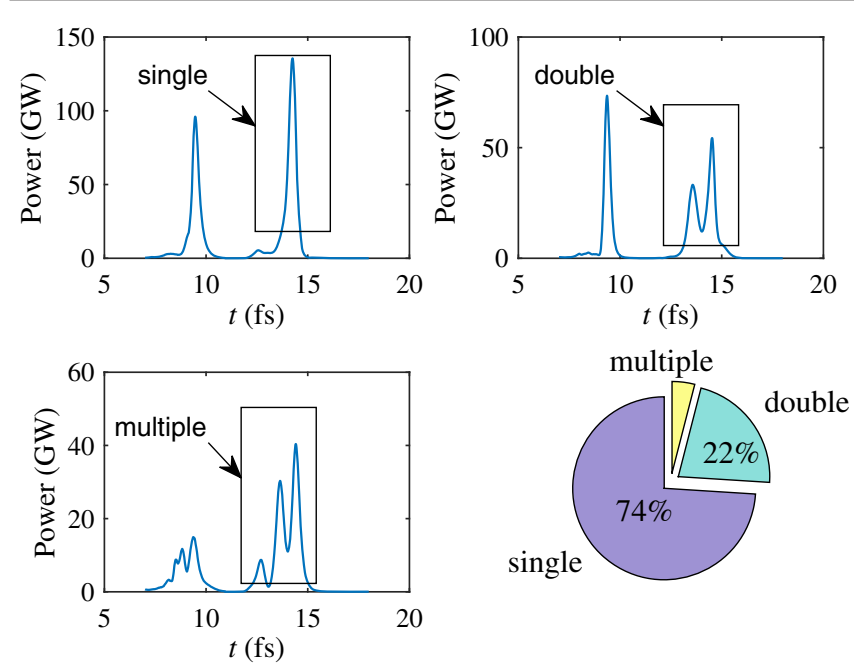

FIG. 5. Examples of simulated FEL pulses with single, double and multiple spikes in the power profile. The pie shows the proportion of these three shapes. An additional 5 fs delay is added to the second-color.
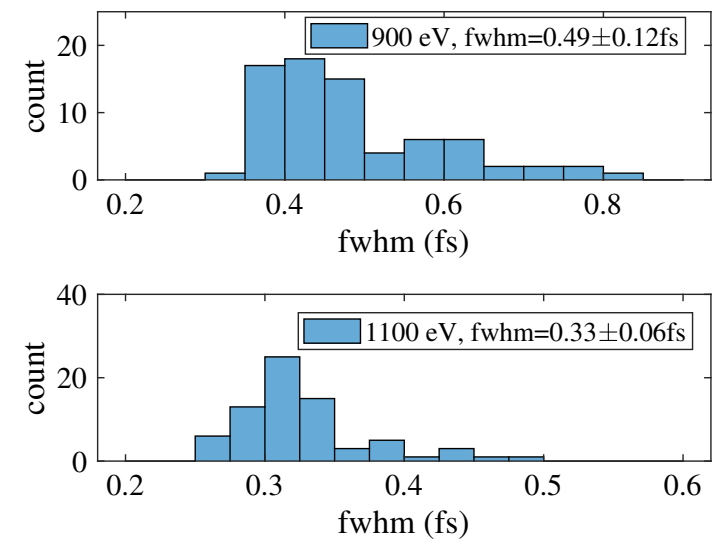

FIG. 6. Distribution of the FWHM of $900 \mathrm{eV}$ (top) and $1100 \mathrm{eV}$ (bottom) pulses.
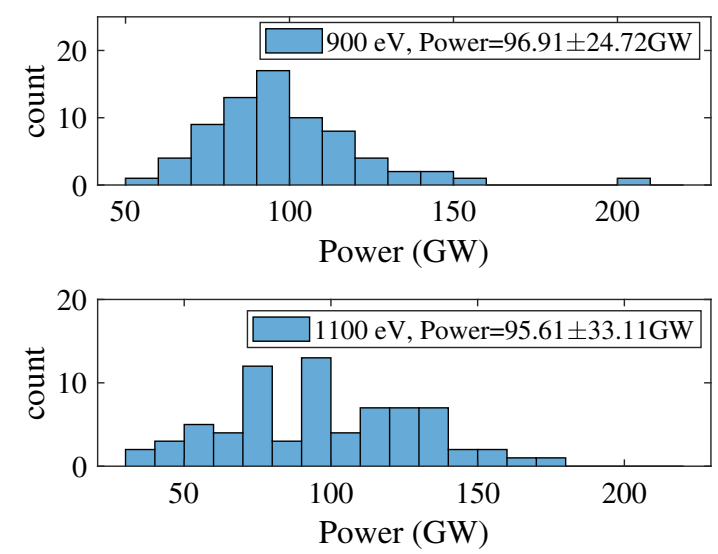

FIG. 7. Distribution of the peak power of $900 \mathrm{eV}$ (top) and $1100 \mathrm{eV}$ (bottom) pulses.

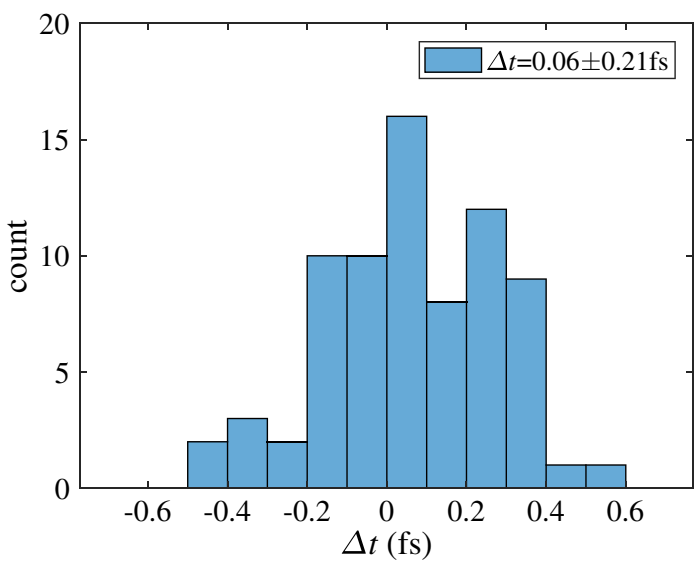

FIG. 8. Distribution of the time separation between pairs of pulses.

100 shots with different initial random seeds in the GENESIS simulation. It can be seen that with the taper settings in Fig. 3, 74 of 100 shots are of single-spike profile for both colors and 22 of 100 shots have at least one double-spike shape. In the simulation, most of the double-spike pulses appear in the first color where the undulator strength gradually increases. Only 4 of 100 shots have three or more spikes in the power profile.

For the simulation outputs with single-spike power profile, the distribution of the full-width-of-half-maximum (FWHM) of each pulse are given in Figs. 6. The FWHM of the single-spike pulses is $0.49 \pm 0.12$ fs for the first color $(900 \mathrm{eV})$, and $0.33 \pm 0.06$ fs for the second color. It can be observed from the distribution that the FWHMs of most

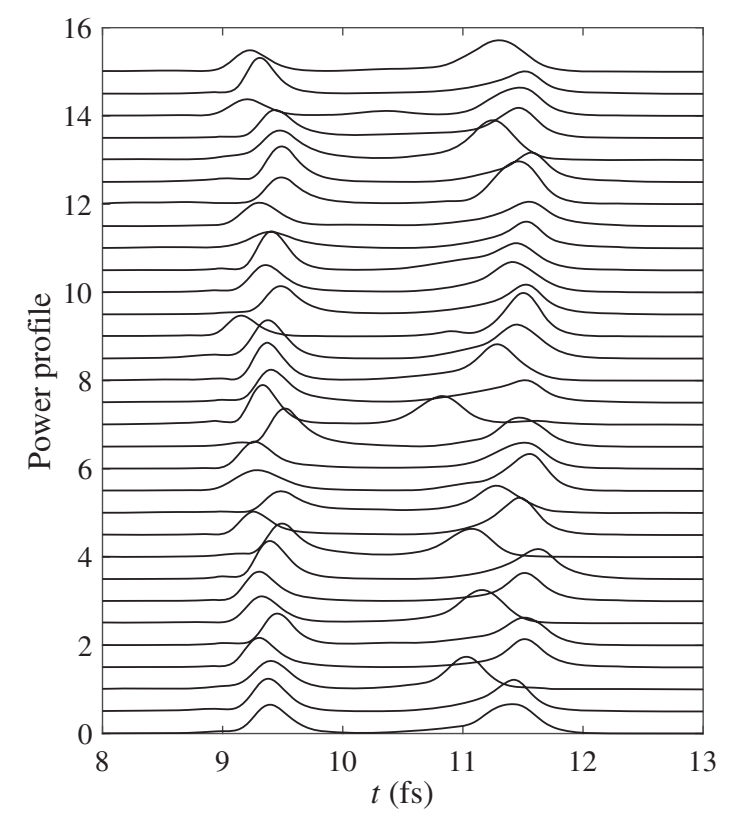

FIG. 9. Typical power profiles of the single-spike two-color FEL pulses. An additional 2 fs delay from the chicane is added to the second color (the left) pulse. 

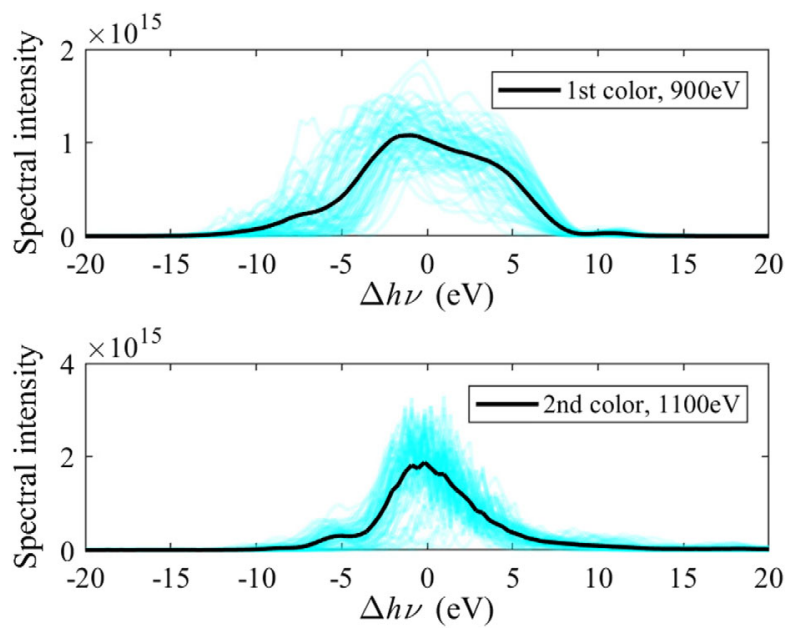

FIG. 10. Spectra of the two-color FELs. The solid black line is the averaged spectra over the single-spike shots shown in blue.

shots are centered around $0.4 \mathrm{fs}$. The distribution of the peak power of the two-color pulses are presented in Fig. 7. The average power of the two pulses are both about $96 \mathrm{GW}$.

The time delay of the two pulses can be calculated by the longitudinal separation of the centroid of the two pulses. The distribution of the time delay of the single-spike shots is given in Fig. 8. When the delay of the magnetic chicane between the two undulator sections is zero, the time delay of the two pulses is $0.06 \mathrm{fs}$, meaning that the two pulses almost overlap together at the end of the undulators. Additional delay can be added by the chicane to vary the delay from 0 to tens of or more than one hundred of fs continuously. The jitter of the time delay is about $0.21 \mathrm{fs}$. The power profile of 30 typical pulses are shown in Fig. 9. To illustrate the two pulses clearly, an additional 2 fs time delay from the chicane is added to the second pulses. It can be seen that the main source of the time delay jitter comes from the pulse position jitter of the first color.

In addition to the power profile, we also provide the spectra of the two-color FELs, as shown in Fig. 10. The solid black line in the figures is the spectra averaged over all shots whose power profiles are single-spike shape. The FWHM bandwidth is about $10 \mathrm{eV}$ for $900 \mathrm{eV}$ FEL pulses
TABLE II. Parameters of self-modulation scheme.

\begin{tabular}{lcc}
\hline \hline Parameter & Value & Unit \\
\hline Wiggler period $\lambda_{w}$ & 35 & $\mathrm{~cm}$ \\
Wiggler strength $K$ & 46.4 & \\
Resonant wavelength $\lambda_{r}$ & 2 & $\mu \mathrm{m}$ \\
Number of periods in 1st stage & 18 & \\
Number of periods in 2nd stage & 24 & \\
Four-dipole chicane $R_{56}$ & -100 & $\mu \mathrm{m}$ \\
\hline \hline
\end{tabular}

and $7 \mathrm{eV}$ for $1100 \mathrm{eV}$ pulses, corresponding to timebandwidth product of 2.6 and 1.2. The pulses of $1100 \mathrm{eV}$ are close to the Fourier transform limit while there are some residual chirps on the $900 \mathrm{eV}$ pulses.

\section{START-TO-END SIMULATION}

In this section, the simulation results of the two-color FEL with a start-to-end beam are presented. We use the nominal LCLS-II copper linac beamline parameters to produce an electron beam with the same energy in Table I. In order to generate the energy modulation in the electron beam, we take advantage of the self-modulation effect of the electron beam in a wiggler $[13,14]$. In the self-modulation process, the coherent radiation field generated from the beam tail modulates the slices of electron beam in front of it. To maximize the energy modulation amplitude, we propose a new two-stage self-modulation scheme, which is illustrated in Fig. 11. In the first-stage, the radiation from the tail of the bunch generates a small energy modulation in the core. The energy modulation is converted into density bunching by a magnetic chicane. The second-stage wiggler amplifies the initial energy modulation using the short-term coherent synchrotron radiation (CSR) wake from the existing bunching. The parameters of the wiggler and chicane for the selfmodulation are shown in Table II. For the nominal LCLS-II copper linac beam after compression, as shown in Fig. 12(a), the current horn is usually overcompressed, which is not suitable for the self-modulation. To increase the peak current and available wiggler period number, we can vary the $R_{56}$ of the second dog-leg (DL2) in the LCLS-II beamline which is

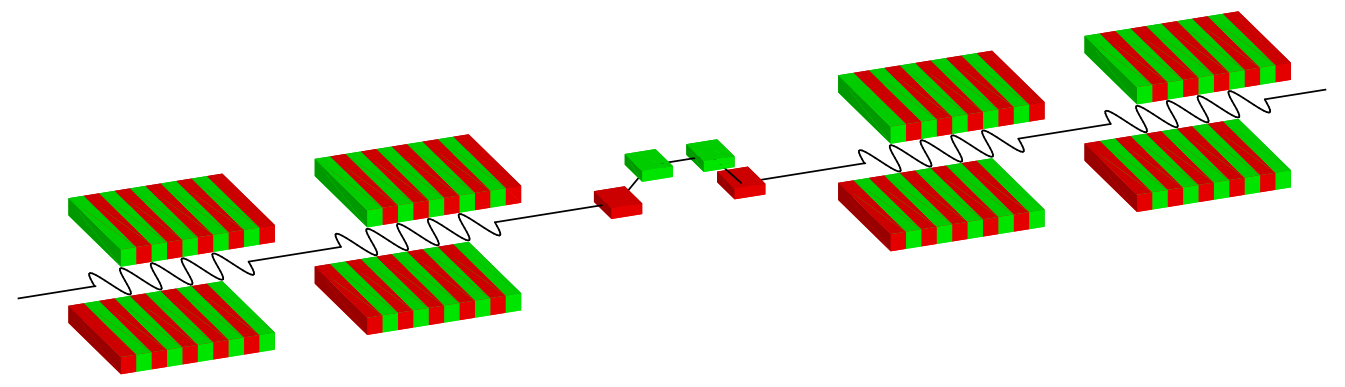

FIG. 11. Layout of the two-stage self-modulation scheme for the generation of two-color FELs, including the first-stage wiggler to modulate the beam with the radiation field from the current horn, the magnetic chicane to convert energy modulation to density modulation and the second-stage wiggler to amplify the modulation. 

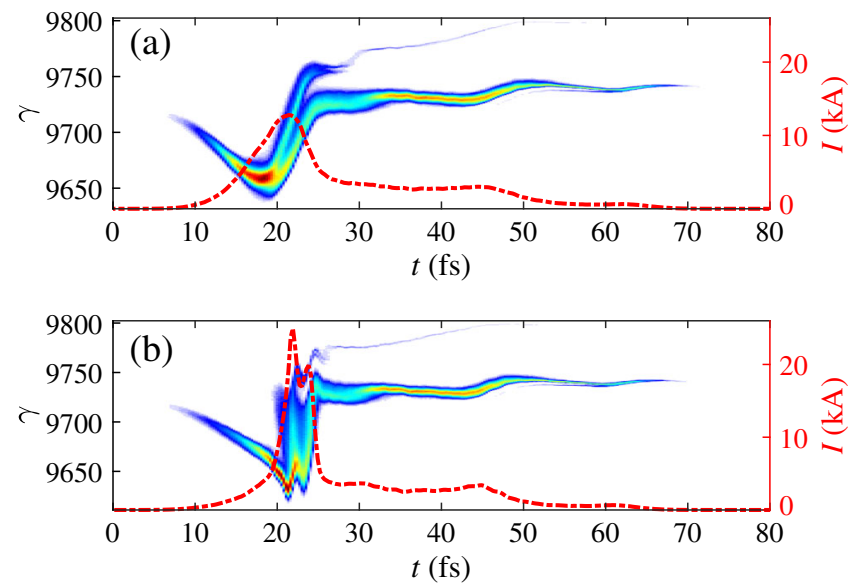

FIG. 12. Longitudinal phase space of the electron beam at the beginning of the self-modulation wiggler with different $R_{56}$ of DL2: (a) $R_{56}=0$ and (b) $R_{56}=200 \mu \mathrm{m}$. The red dotted line is the current profile along the beam. The beam's head is to the right.

located between the linac and undulator tunnel, optimizing the phase space of the current horn. The $R_{56}$ of the DL2 can be varied from $-5 \mathrm{~mm}$ to $5 \mathrm{~mm}$ with a nominal value of zero. This contrasts with the $R_{56}$ of a four-dipole chicane which is negative. Figure 12(b) presents the longitudinal phase space of the beam with DL2 $R_{56}=200 \mu \mathrm{m}$. The current horn is
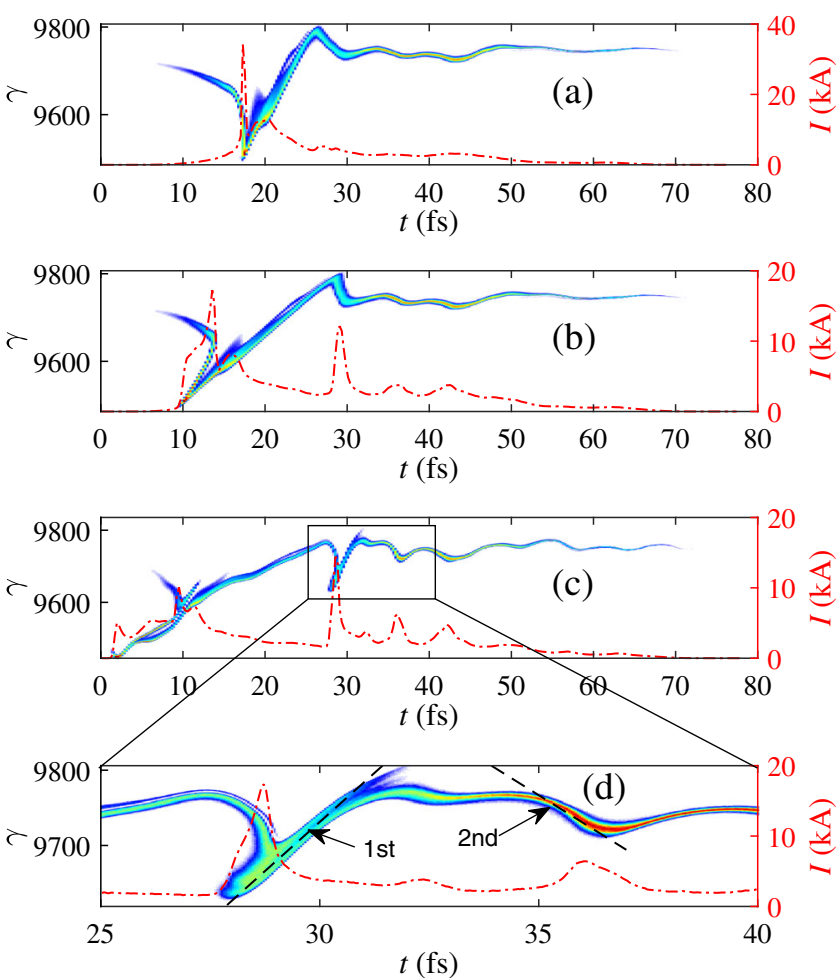

FIG. 13. Longitudinal phase space of the electron beam (a) after the first-stage wiggler, (b) after the magnetic chicane and (c) after the second-stage wiggler. The enlarged phase space from figure (c) is given in figure (d) with the energy chirp chosen for the twocolor FELs. The beam's head is to the right. compressed by the DL2 to be more than $20 \mathrm{kA}$, which enhances the self-modulation.

For the simulation of the self-modulation, we employ the model of the CSR impedance from a wiggler developed in Refs. $[13,14]$. Figure 13 shows the evolution of electron beam phase space in the self-modulation process, including the longitudinal phase space after (a) the first-stage wiggler, (b) the magnetic chicane, and (c) the second-stage wiggler. In Fig. 13(d), the enlarged phase space after modulation is given to illustrate the energy chirp which are chosen for the two-color FEL generation. Compared with the ideal case, the beam is not uniform in current and transverse emittance and the chosen lasing parts have higher current, which is helpful to suppress lasing from undesired parts. We will assume that lasing in the head of the bunch is suppressed by either an emittance spoiler or an energy spoiler.

The double chirp-taper scheme is optimized using the same procedure discussed in the previous section. The optimal taper coefficient $d K /(K d z)$ for the first section is $1.5 \times 10^{-3}$. The taper of the second section is $d K /(K d z)=$ $-1.5 \times 10^{-3}$ and the last three undulators have constant strength, which is intended to further amplify the pulse after saturation. The required taper range can be achieved by the variable-gap undulators in the LCLS-II.

Using the optimized undulator taper in Fig. 14, 100 runs of GENESIS simulation were performed with different beam shot noise. Due to the higher peak current at the chosen lasing parts in the start-to-end beam, the ratio of singlespike pulses is higher than that of the ideal beam, up to $86 \%$. The statistical results of the FWHM and peak power of these single-spike shots are shown in Fig. 15. The FWHM of the two pulses are both about $0.4 \pm 0.1 \mathrm{fs}$. The average peak power over 100 shots of the pulse generated in the first undulator section is $168 \mathrm{GW}$, which is higher than the peak power of the second pulse (107 GW). The higher peak power is a result of the larger peak current in this situation.
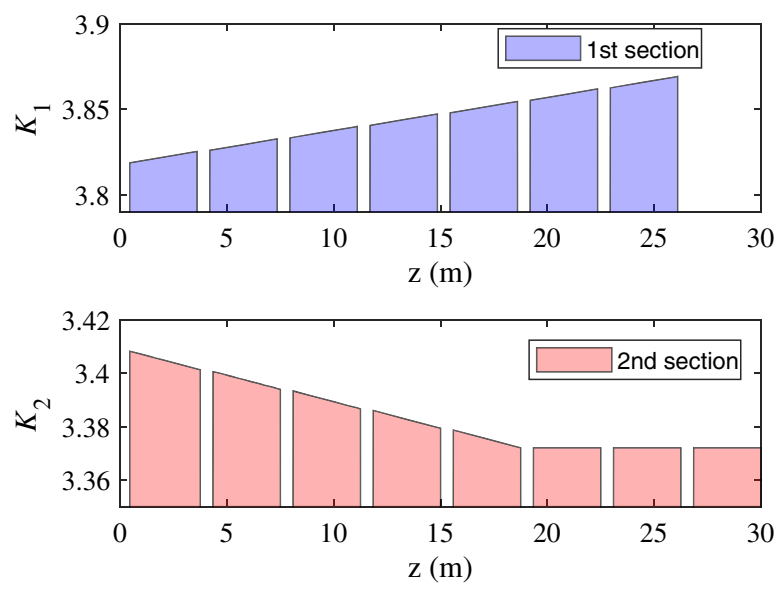

FIG. 14. The optimized undulator strength taper of the two sections for the generation of two-color FELs. 

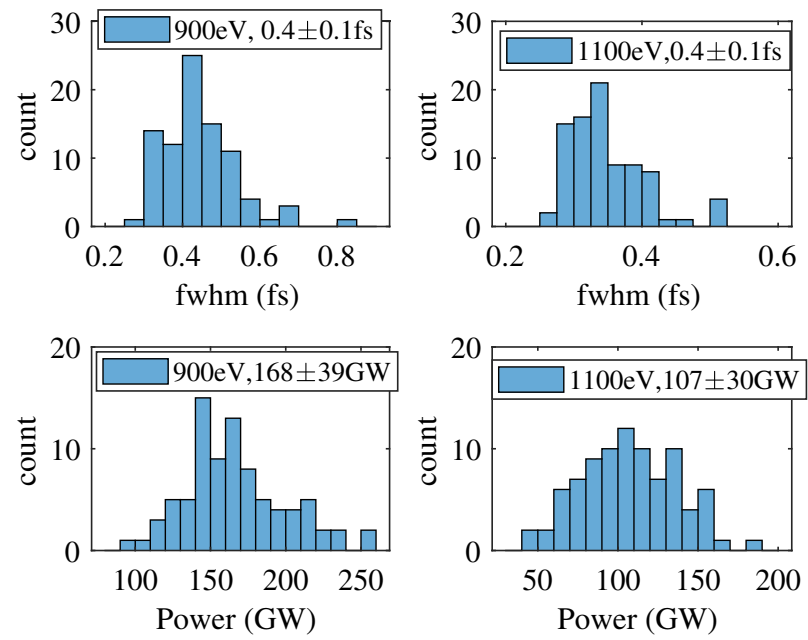

FIG. 15. Distribution of the FWHM (top row) and peak power (bottom row) of the two-color FEL pulses.

The time separation between the two pulses is presented in Fig. 16. When there is no additional delay from the magnetic chicane, the average time delay is about $4.82 \mathrm{fs}$ with $0.19 \mathrm{fs}$ jitter. As the delay from the chicane is increased, the time delay between the two pulses will first come to zero and then increase again to the required value. The maximum delay is limited by the longitudinal dispersion of the delay stage. As the electrons are delayed, they are also compressed or decompressed depending on the sign of the energy-chirp. This effect is negligible if

$$
2\left|\Delta t_{\text {chicane }} \frac{d \gamma}{\gamma d t}\right| \ll 1
$$

In principle even if this condition is violated, one could rearrange the taper to compensate for the change in chirp.

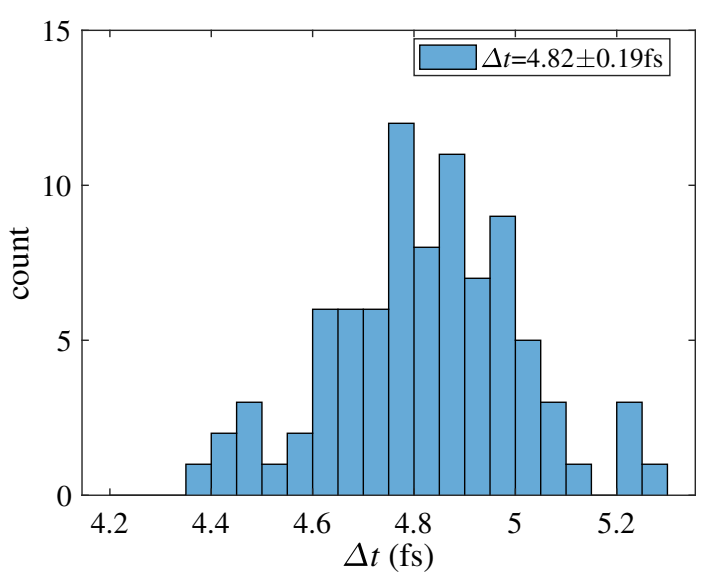

FIG. 16. Distribution of the absolute value of the time delay of the two-color XFEL pulses. The first color is behind the second when the chicane delay is zero.

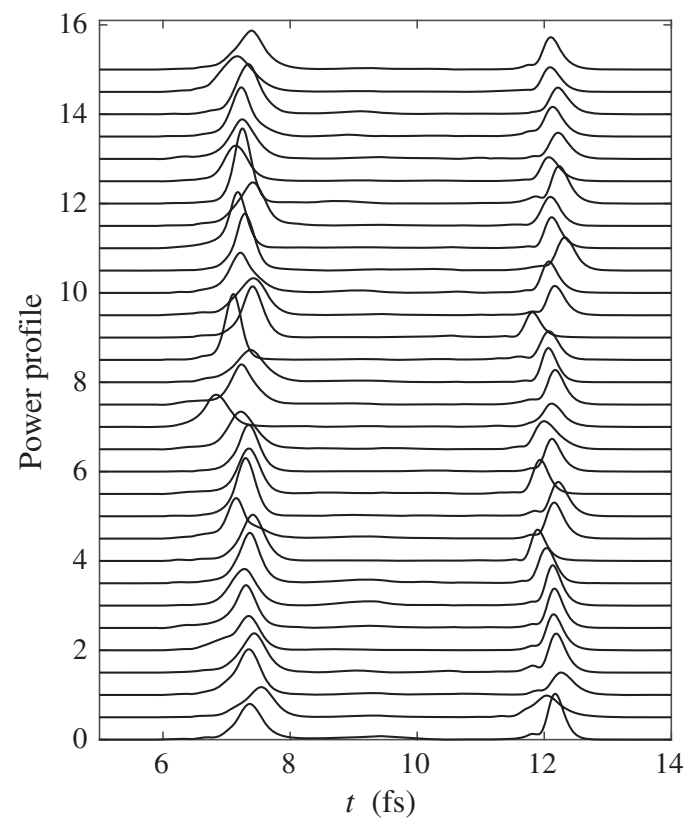

FIG. 17. Typical power profiles of the single-spike two-color FEL pulses from the start-to-end electron beam.

However in the interest of preserving the pulse properties it is better to operate under the condition of negligible compression. For the beam parameters used in this paper the maximum delay is of order $100 \mathrm{fs}$.

30 shots of typical power profiles are presented in Fig. 17 and the average spectra are shown in Fig. 18, respectively. Similar with the case of ideal beam, the jitter of the time delay mostly comes from the timing jitter of the first color. The FWHM bandwidth of the $900 \mathrm{eV}$ pulses is $\sim 5 \mathrm{eV}$ and the time-bandwidth product is 1.1 . For the $1100 \mathrm{eV}$ pulses, the FWHM bandwidth is $\sim 6 \mathrm{eV}$ and the timebandwidth product is 1.2. Both of the two colors are close to the Fourier transform limit.
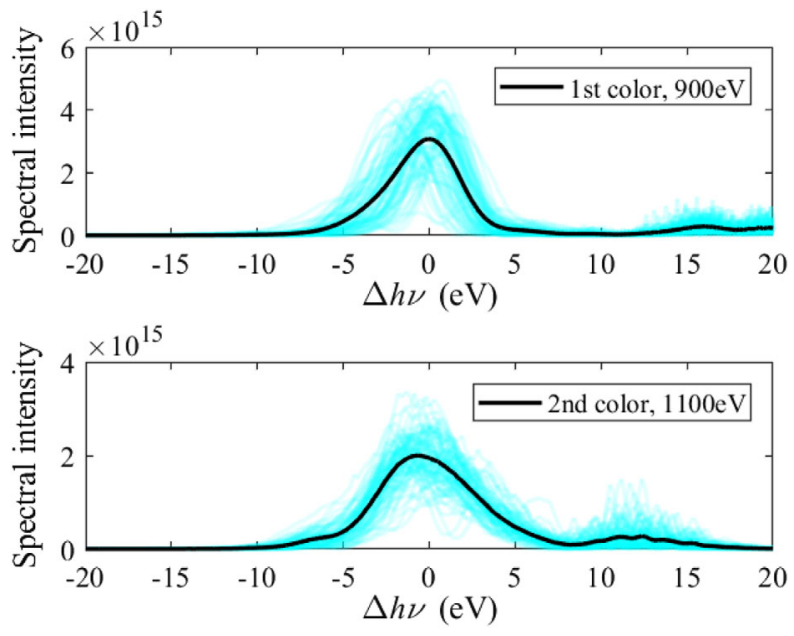

FIG. 18. Spectra of the two-color FELs. The solid black line is the averaged spectra over the single-spike shots shown in blue. 


\section{DISCUSSION}

The method we propose enables sub-fs time resolution with full saturation power, full control of the photon energy (limited by the undulator tuning range) and the ability to scan the delay through the overlap condition. Compared to other multiundulator two-color schemes [7-9] the proposed method is similar to the fresh-slice method [10]: in both schemes the two colors are generated in two different undulators using two different regions of an electron bunch, which results in higher peak power and improved time separation range.

With respect to the fresh-slice method based on the dechirper, the use of a modulated electron bunch improves the timing jitter of the two pulses. This is because in the double chirp-taper scheme the separation of the two lasing slices is given by half of the modulation wavelength, which in turn is locked to the resonant condition in the wiggler, effectively decoupling the time separation from the peak current jitter of the electron bunch. In the fresh-slice scheme, instead, the jitter in the bunch duration is directly translated into timing jitter between the two x-ray pulses. For example, data reported in [13] shows a wavelength jitter of $3 \%$ for the self-modulation process using an electron beam with a $10 \%$ bunch duration jitter. This additional jitter is comparable to the intrinsic jitter of the two pulses observed in this paper and does not significantly affect the ability to resolve sub-fs dynamics with the proposed scheme. Furthermore, high-repetition rate FELs powered superconducting $\mathrm{CW}$ linacs are expected to have significantly reduced peak current jitter [1], further suppressing this additional source of timing jitter.

Finally, due to the use of a passive modulator this method is directly applicable to the next generation of highrepetition rate FELs based on superconducting accelerators.

\section{SUMMARY}

In this paper we proposed a method to produce pairs of sub-fs XFEL pulses with individually controllable color and time separation based on a double chirp-taper scheme. An electron beam is modulated in a two-stage selfmodulation scheme. The undulator is divided in two parts and the taper of the each undulator section is optimized to match the energy chirp in two distinct regions of the bunch. GENESIS simulations have been performed with an ideal beam with a sinusoidal energy modulation and using a start-to-end beam simulation of the LCLS-II copper linac beam. The proposed method can generate two sub-fs single-spike XFEL pulses individually tunable in energy. The time-delay can be varied arbitrarily using a magnetic chicane in between the two tapered undulators. The FWHM duration of each pulse is $\sim 0.4 \mathrm{fs}$ with tunable time delay from zero to roughly $100 \mathrm{fs}$. The arrival time jitter of the two pulses is less than $0.2 \mathrm{fs}$ and the peak power can reach $\sim 100 \mathrm{GW}$. The use of the passive modulator makes this scheme suitable for high-repetition rate machines. The proposed method provides a powerful tool for attosecond pump-probe experiments at x-ray FEL facilities.

\section{ACKNOWLEDGMENTS}

We would like to thank James P. Cryan and Alexander Zholents for useful discussions. This work was supported by U.S. Department of Energy Contracts No. DE-AC0276SF00515, DOE-BES Accelerator and detector research program Field Work Proposal 100317 and Department of Energy, Laboratory Directed Research and Development program at SLAC National Accelerator Laboratory, under Contract No. DE-AC02-76SF00515.

[1] R. Schoenlein, P. Abbamonte, F. Abild-Pedersen, P. Adams, M. Ahmed, F. Albert, R. Alonso Mori, A. Anfinrud, A. Aquila, M. Armstrong et al., New science opportunities enabled by lcls-ii x-ray lasers, SLAC Report No. SLAC-R-1053, 2015.

[2] T. Heinz, O. Shpyrko, D. Basov, N. Berrah, P. Bucksbaum, T. Devereaux, D. Fritz, K. Gaffney, O. Gessner, V. Gopalan, Z. Hasan et al., Opportunities for basic research at the frontiers of xfel ultrafast science, Report of the Basic Energy Sciences Roundtable, 2017, https://science.energy .gov/ /media/bes/pdf/reports/2018/Ultrafast_x-ray_ science_rpt.pdf.

[3] F. Calegari, D. Ayuso, A. Trabattoni, L. Belshaw, S. De Camillis, S. Anumula, F. Frassetto, L. Poletto, A. Palacios, P. Decleva et al., Ultrafast electron dynamics in phenylalanine initiated by attosecond pulses, Science 346, 336 (2014).

[4] E. Goulielmakis, Z.-H. Loh, A. Wirth, R. Santra, N. Rohringer, V.S. Yakovlev, S. Zherebtsov, T. Pfeifer, A. M. Azzeer, M. F. Kling et al., Real-time observation of valence electron motion, Nature (London) 466, 739 (2010).

[5] J. Breidbach and L. S. Cederbaum, Universal Attosecond Response to the Removal of an Electron, Phys. Rev. Lett. 94, 033901 (2005).

[6] F. Remacle and R. D. Levine, An electronic time scale in chemistry, Proc. Natl. Acad. Sci. U.S.A. 103, 6793 (2006).

[7] A. A. Lutman, R. Coffee, Y. Ding, Z. Huang, J. Krzywinski, T. Maxwell, M. Messerschmidt, and H-D. Nuhn, Experimental Demonstration of Femtosecond TwoColor X-ray Free-Electron Lasers, Phys. Rev. Lett. 110, 134801 (2013).

[8] T. Hara, Y. Inubushi, T. Katayama, T. Sato, H. Tanaka, T. Tanaka, T. Togashi, K. Togawa, K. Tono, M. Yabashi et al., Two-colour hard X-ray free-electron laser with wide tunability, Nat. Commun. 4, 2919 (2013).

[9] A. Marinelli, A. A. Lutman, J. Wu, Y. Ding, J. Krzywinski, H.-D. Nuhn, Y. Feng, R. N. Coffee, and C. Pellegrini, Multicolor Operation and Spectral Control in a GainModulated X-ray Free-Electron Laser, Phys. Rev. Lett. 111, 134801 (2013).

[10] A. A. Lutman, T. J. Maxwell, J. P. MacArthur, M. W. Guetg, N. Berrah, R. N. Coffee, Y. Ding, Z. Huang, A. 
Marinelli, S. Moeller et al., Fresh-slice multicolour x-ray free-electron lasers, Nat. Photonics 10, 745 (2016).

[11] A. Marinelli, D. Ratner, A. A. Lutman, J. Turner, J. Welch, F.-J. Decker, H. Loos, C. Behrens, S. Gilevich, A. A. Miahnahri et al., High-intensity double-pulse x-ray freeelectron laser, Nat. Commun. 6, (2015).

[12] K. R. Ferguson, M. Bucher, T. Gorkhover, S. Boutet, H. Fukuzawa, J. E. Koglin, Y. Kumagai, A. Lutman, A. Marinelli, M. Messerschmidt et al., Transient lattice contraction in the solid-to-plasma transition, Sci. Adv. 2, e1500837 (2016).

[13] J. MacArthur, J. Duris, Z. Huang, A. Marinelli, and Z. Zhang, Self-modulation of a relativistic electron beam in a wiggler, in 9th International Particle Accelerator Conf. (IPAC'18), Vancouver, BC, Canada, 2018, (JACOW Publishing, Geneva, Switzerland, 2018), p. 4492-4495.

[14] J. P. MacArthur, J. Duris, Z. Zhang, Z. Huang, and A. Marinelli, Phase-stable self-modulation of an electron beam in a wiggler (to be published).

[15] E. L. Saldin, E. A. Schneidmiller, and M. V. Yurkov, Terawatt-scale sub-10-fs laser technology-key to generation of gw-level attosecond pulses in x-ray free electron laser, Opt. Commun. 237, 153 (2004).

[16] E. L. Saldin, E. A. Schneidmiller, and M. V. Yurkov, A new technique to generate $100 \mathrm{gw}$-level attosecond $\mathrm{x}$-ray pulses from the X-ray sase fels, Opt. Commun. 239, 161 (2004).

[17] E. L. Saldin, E. A. Schneidmiller, and M. V. Yurkov, Selfamplified spontaneous emission fel with energy-chirped electron beam and its application for generation of attosecond x-ray pulses, Phys. Rev. ST Accel. Beams 9, 050702 (2006).

[18] A. A. Zholents and M. S. Zolotorev, Attosecond X-ray pulses produced by ultra short transverse slicing via laser electron beam interaction, New J. Phys. 10, 025005 (2008).

[19] Y. Ding, Z. Huang, D. Ratner, P. Bucksbaum, and H. Merdji, Generation of attosecond x-ray pulses with a multicycle two-color enhanced self-amplified spontaneous emission scheme, Phys. Rev. ST Accel. Beams 12, 060703 (2009).

[20] L. Giannessi, A. Bacci, M. Bellaveglia, F. Briquez, M. Castellano, E. Chiadroni, A. Cianchi, F. Ciocci, M. E. Couprie, L. Cultrera et al., Self-amplified Spontaneous
Emission Free-Electron Laser with an Energy-Chirped Electron Beam and Undulator Tapering, Phys. Rev. Lett. 106, 144801 (2011).

[21] S. Krinsky and Z. Huang, Frequency chirped self-amplified spontaneous-emission free-electron lasers, Phys. Rev. ST Accel. Beams 6, 050702 (2003).

[22] W. M. Fawley, Production of ultrashort fel xuv pulses via a reverse undulator taper, Nucl. Instrum. Methods Phys. Res., Sect. A 593, 111 (2008).

[23] P. Baxevanis, J. Duris, Z. Huang, and A. Marinelli, Timedomain analysis of attosecond pulse generation in an X-ray free-electron laser, Phys. Rev. Accel. Beams 21, 110702 (2018).

[24] S. Reiche, Genesis 1.3: A fully 3d time-dependent fel simulation code, Nucl. Instrum. Methods Phys. Res., Sect. A 429, 243 (1999).

[25] P. Emma, K. Bane, M. Cornacchia, Z. Huang, H. Schlarb, G. Stupakov, and D. Walz, Femtosecond and Subfemtosecond X-ray Pulses from a Self-Amplified SpontaneousEmission-Based Free-Electron Laser, Phys. Rev. Lett. 92, 074801 (2004).

[26] Y. Ding, C. Behrens, R. Coffee, F.-J. Decker, P. Emma, C. Field, W. Helml, Z. Huang, P. Krejcik, J. Krzywinski et al., Generating femtosecond $\mathrm{X}$-ray pulses using an emittancespoiling foil in free-electron lasers, Appl. Phys. Lett. 107, 191104 (2015).

[27] A. Marinelli, R. Coffee, S. Vetter, P. Hering, G. N. West, S. Gilevich, A. A. Lutman, S. Li, T. Maxwell, J. Galayda et al., Optical Shaping of X-ray Free-Electron Lasers, Phys. Rev. Lett. 116, 254801 (2016).

[28] J. Duris, Z. Huang, J. P. MacArthur, and A. Marinelli, Superradiant amplication in a chirped-tapered $\mathrm{X}$-ray freeelectron laser (to be published).

[29] R. Bonifacio, B. W. J. McNeil, and P. Pierini, Superradiance in the high-gain free-electron laser, Phys. Rev. A 40, 4467 (1989).

[30] R. Bonifacio, N. Piovella, and B. W. J. McNeil, Superradiant evolution of radiation pulses in a free-electron laser, Phys. Rev. A 44, R3441 (1991).

[31] K.-J. Kim, Z. Huang, and R. Lindberg, Synchrotron Radiation and Free-Electron Lasers (Cambridge University Press, Cambridge, England, 2017). 